\title{
Polarization of the Graphene-Liquid Electrolyte Interface Probed by SEM
}

Andrei Kolmakov ${ }^{1}$, Hongxuan Guo ${ }^{1,2}$, Alexander Yulaev ${ }^{1,2}$, Evgheni Strelcov ${ }^{1,2}$ and Alexander $\mathrm{Tselev}^{3}$

1. Center for Nanoscale Science and Technology, NIST, Gaithersburg, MD.

2. Maryland Nano Center, University of Maryland, College Park, MD.

3. CICECO and Department of Physics, University of Aveiro, Aveiro, Portugal

The formation and properties of the electrical double layer (EDL) at the solid-liquid electrolyte interface are crucial for electrochemical devices e.g. supercapacitors, batteries, sensors as well as for biomedical applications. Various techniques are employed to investigate the structure and composition of the EDL, which are mainly based on optical methods (see review [1]) and more recently on electron spectroscopy $[2,3]$. Many applications, however, require real-time probing of liquid interfaces at (meso-) and nanoscale. The latter remains to be an experimentally challenging task.

Recently we developed a graphene-capped micro-channels array platform (MCA) for characterization of liquid samples and aqueous solutions under UHV using standard surface science techniques [4]. Using this MCA platform, we studied the pure water and electrochemical reaction in aqueous electrolytes, such as $\mathrm{CuSO} 4$ with different electron imaging and spectroscopy techniques. [4-6]

In this study, we found that secondary electron (SE) yield from graphene membrane on the top of electrolyte sensitively depends on the MCA cell polarization potential. The SE depends on the electron density of states in the graphene and within EDL as well as the local potential inside the electron escape depth region. With the increase of the polarization potential at the bottom electrode, the secondary electron yield decreases due to the redistribution of the potential inside electrolyte, double layers and graphene membrane (Fig. 1). As a reference sample, graphene membrane on the top of toluene was measured. We found that the secondary electron yield is stable since the ion concentration in the toluene is negligible to form appreciable EDL. The relationship between normalized SE yields and applied potential for these two cases is shown in the Fig. 2a. To support experimental observations, the effect of applied bias to the EDL formation and graphene screening was numerically simulated using finite elements (FE) modeling (Fig. 2b).

In summary, we developed a new in situ method to probe the evolution of the EDL at the grapheneliquid interface during polarization conditions using standard scanning electron microscope. This work will impact the characterization of the electrochemical materials and devices under real-world operation conditions.

References:

[1] F. Zaera, Chem. Rev. 112 (2012), p. 2920.

[2] M. Favaro et al, Nature Communications 7 (2016), p. 12695.

[3] M. A. Brown et al, Physical Reviews X 6 (2016), p. 011007.

[4] A. Yulaev et al, ACS Applied Materials \& Interfaces 9 (2017), p. 26492.

[5] H. Guo et al, Nano Letters 17 (2017), p. 1034.

[6] S. Nemšák et al, JACS 139 (2017), p. 18138. 
a)

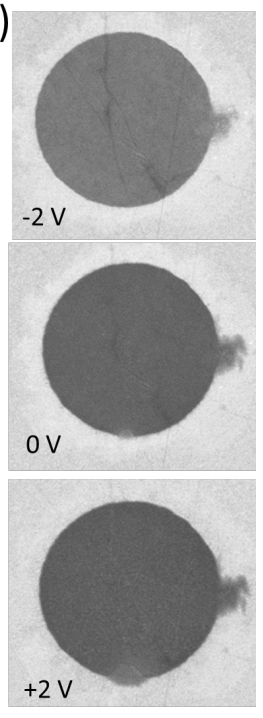

b)

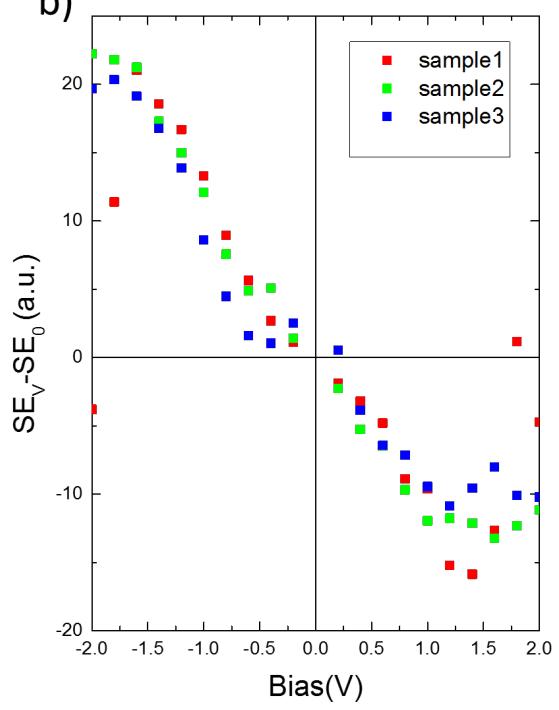

Figure 1. (a) SEM images of $0.1 \mathrm{M} \mathrm{CuSO}_{4}$ electrolyte under grounded graphene membrane under different bias voltage at opposite electrode. (d) The difference between SE yields of the biased and grounded graphene-electrolyte interface as a function of the cell polarization.

a)

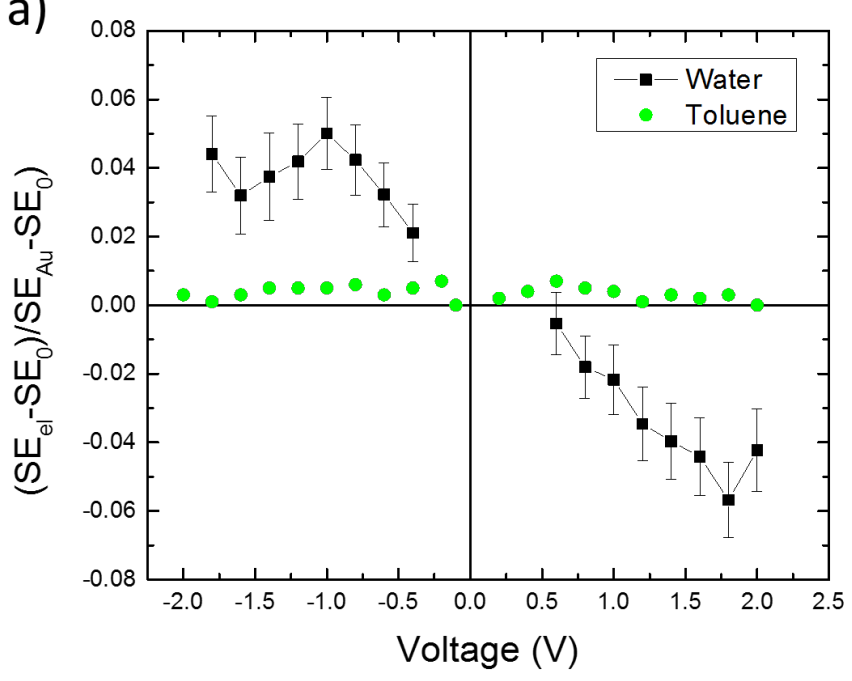

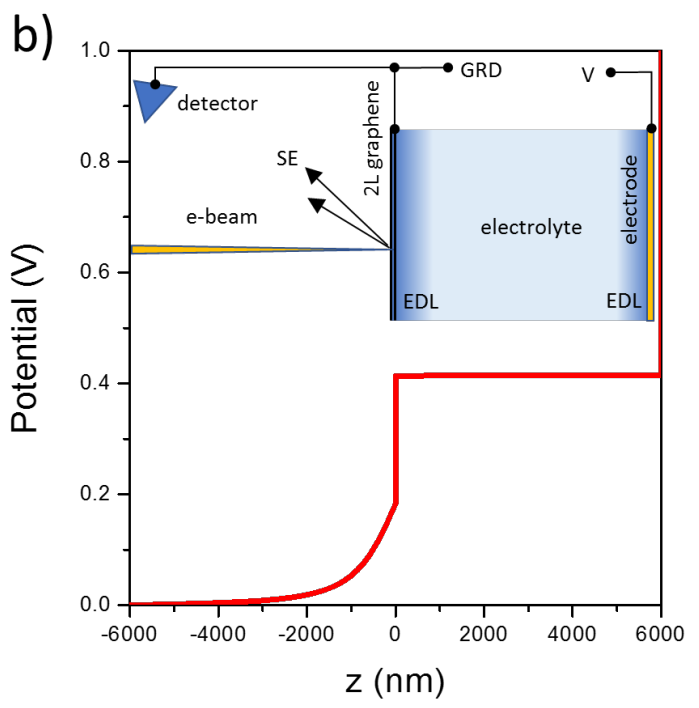

Figure 2. (a) Normalized SE yields from the graphene capped water and toluene as a function of cell polarization. (b) Numerical simulation of the electric field distribution across $1 \mathrm{~V}$ biased liquid MCA cell using FE modeling 